\title{
Efficacité comparée de l'urée et du biuret dans des régimes distribués en 2 ou 6 fois par jour, chez le mouton
}

\author{
J. GONZALEZ, Carmen LOPEZ, Maria R. ALVIR, J.F. GALVEZ \\ Departamento de Nutrición Animal, \\ Escuela Técnica Superior de Ingenieros Agrónomos, \\ Universidad Politécnica de Madrid. \\ Ciudad Universitaria, 28040 Madrid (Espagne)
}

\begin{abstract}
Résumé
Après 5 semaines d'adaptation, 2 lots de 3 agneaux munis d'une canule du rumen ont été employés pour évaluer l'utilisation de 2 aliments composés complémentés avec de l'urée (U) ou du biuret (B), et distribués, au cours de deux périodes successives, en 2 ou 6 repas/jour.

Les coefficients d'utilisation digestive (CUD), les bilans azotés, le $\mathrm{pH}$ et les concentrations d'ammoniac et des acides gras volatils du jus de rumen ont été déterminés, alors que l'activité cellulolytique de celui-ci a été estimée par les coefficients de disparition in sacco de "l'acid detergent fibre" (ADF) d'échantillons de paille broyée.

Les concentrations d'ammoniac du jus du rumen ont été significativement plus faibles $(P<0,05)$ avec l'aliment $B$ qu'avec l'aliment $U$. L'augmentation du nombre de repas quotidiens, de 2 à 6 a entraîné une baisse des maximas de concentrations d'ammoniac, plus marquée avec l'aliment $U$ qu'avec l'aliment $B$. De même, cette augmentation a amélioré $(P<0,05)$ le bilan azoté avec l'aliment $\mathrm{U}(3,84$ et $5,93 \mathrm{~g} \mathrm{~N}$ retenu/animal/jour, avec 2 et 6 repas/jour, respectivement), tandis qu'aucun effet n'était observé avec l'aliment B $(5,42$ et $5,31 \mathrm{~g} \mathrm{~N}$ retenu/animal/jour, avec 2 et 6 repas/jour. respectivement).

Les CUD de la matière organique, des matières azotées et de l'ADF ne sont généralement pas modifiés sauf pour le CUD de l'ADF qui a été plus faible $(\mathrm{P}<0,01)$ avec $2(28,3$ p. 100) qu'avec $6(34,3$ p. 100$)$ repas/jour. De plus, avec cette dernière fréquence d'alimentation. l'activité cellulolytique dans le rumen a été moindre $(P<0,01)$ avec l'aliment $U(36,1$ p. 100) qu'avec laliment B (54,5 p. 100).
\end{abstract}

Mots clês : Urée, biuret, fréquence d'alimentation, ovins.

\section{Introduction}

Parmi les sources d'azote non protéique d'origine industrielle (ANPI), la plus utilisée est l'urée. Cependant, son emploi présente un certain nombre d'inconvénients, en raison de sa vitesse de dégradation très élevée dans le rumen. Ainsi, lorsqu'on 
emploie de fortes doses d'urée et, tout particulièrement quand on introduit celle-ci dans des rations riches en glucides lentement fermentescibles (polysaccharides - $\beta$ ), il peut se produire une accumulation ruminale d'ammoniac qui entraîne le plus souvent une résorption de fortes quantités de ce métabolite. Il en résulte une baisse de l'efficacité d'utilisation de l'azote et, dans certains cas, des effets toxiques (Wolter, 1974 ; INRA, 1978).

Ces inconvénients risquent peu de se manifester si l'on recourt à des composés, tels que le biuret, dont la dégradation dans le rumen est plus lente. Ce produit ne présenterait aucun risque de toxicité (Clark, Barret \& Kellerman, 1963) et son efficacité (après une période d'adaptation suffisante) est élevée et semblable à celle de l'urée (revue de Fonnesbeck, Kearl \& Harris, 1975 ; Singhal \& Mudgal, 1980, 1984) ; cependant, Nikolic et al. (1979) lui attribuent une infériorité, en raison d'une ammoniogénèse trop lente par rapport aux besoins de la population microbienne du rumen.

Le but de ce travail a été d'étudier les effets de l'emploi de l'urée ou du biuret dans les aliments composés sur l'utilisation digestive chez l'agneau à l'engrais, ainsi que l'influence de la fréquence des repas sur l'efficacité de ces deux sources d'ANPI.

\section{Matériel et méthodes}

\section{A. Animaux et schéma expérimental}

Six agneaux (Millschaf $\times$ Manchego) castrés et fistulisés du rumen ont été utilisés. Ces animaux ont été placés dans des cages à métabolisme, répartis au hasard en 2 lots égaux, puis nourris avec deux aliments composés contenant de l'urée (U) ou du biuret (B), avec un temps d'adaptation aux aliments de 5 semaines, afin d'assurer un développement convenable des espèces bactériennes capables d'hydrolyser le biuret (CAmpbell et al., 1963 ; Clemens \& Johnson, 1973). L'âge des animaux au début de la période expérimentale était de 5 mois et le poids moyen pour les deux lots de $34 \pm 1 \mathrm{~kg}$.

La période expérimentale a été divisée en deux sous-périodes successives de 8 jours, pendant lesquelles la ration totale a été distribuée soit en 2 soit en 6 repas égaux par jour, respectivement, à 12 ou à 4 heures d'intervalle. Les deux sous-périodes ont été séparées par une phase de transition de 7 jours. Le niveau d'ingestion a été ajusté dès la $4^{e}$ semaine de la période d'adaptation à $64 \mathrm{~g}$ de $\mathrm{MS} / \mathrm{kg}^{0,75} / \mathrm{jour}$, de manière à n'avoir aucun refus.

Pour chaque fréquence d'alimentation, on a déterminé les coefficients d'utilisation digestive (CUD) de la matière organique (MO), des matières azotées (MA) et de l' « acid detergent fibre » (ADF), le bilan azoté, ainsi que les évolutions du pH et des concentrations en ammoniac et en acides gras volatils (AGV) du jus du rumen. On a également mesuré l'activité cellulolytique de celui-ci, moyennant l'incubation dans le rumen d'échantillons de paille broyée contenue dans des sachets de nylon, pendant un temps de séjour de 48 heures. 


\section{B. Aliments}

On a employé 2 aliments composés complets, présentés en granulés (tabl. 1) ne différant que par l'incorporation soit d'urée soit de biuret (aliments $U$ et $B$, respectivement). La composition centésimale du biuret commercial était la suivante : Biuret 66,7 , urée 18,7, acide cyanurique et triuret 5,1 , eau 5 , agents antiagglomérants et autres 4,5. La teneur en azote de ce produit était de 36,6 p. 100. Les doses d'ANPI ont été déterminées de façon à équilibrer les teneurs en PDI (INRA, 1978) des aliments et représentent 39,25 p. 100 de l'azote total.

\section{TABleau 1}

Composition en matières premières des aliments (p. 100).

Diet ingredients composition (p. 100).

\begin{tabular}{|c|c|c|}
\hline & \multicolumn{2}{|c|}{$\begin{array}{l}\text { Source d'ANPI } \\
N P N \text { compound }\end{array}$} \\
\hline & $\begin{array}{l}\text { Urée } \\
\text { Urea }\end{array}$ & $\begin{array}{l}\text { Biuret } \\
\text { Biuret }\end{array}$ \\
\hline Orge - Barley & 47,35 & 47.13 \\
\hline Paille traitée à la soude - $\mathrm{NaOH}$-treated straw . . . & 33,17 & 33,00 \\
\hline Tourteau de soja ${ }^{(1)}$ - Soyabean meal . . . . . & 7,10 & 7,07 \\
\hline Amidon de maïs - Maize starch $\ldots . .$. & 7,10 & 7,07 \\
\hline Urée - Urea . . . . . . . . . . . . & 1,83 & - \\
\hline Biuret - Biuret $\ldots \ldots \ldots \ldots \ldots \ldots$ & - & 2,28 \\
\hline Complément minéral ${ }^{(2)}$ - Mineral supplement $\ldots$ & 3,45 & 3,45 \\
\hline
\end{tabular}

(1) Chauffé à $80^{\circ} \mathrm{C}$ pendant 24 heures - Heated at $80^{\circ} \mathrm{C}$ for 24 hours.

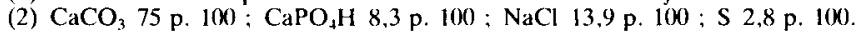

La composition du complément minéral est calculée pour assurer des teneurs en Ca et $P$ dans les aliments de 1 et 0,35 p. 100, respectivement (INRA, 1978), et un rapport $\mathrm{N}: \mathrm{S}$ de 10:1 (Moir, Somers \& Bray, 1967) afin de ne pas limiter le développement de la flore microbienne du rumen, la complémentation en oligoéléments étant réalisée grâce à des pierres à lécher.

La composition chimique des aliments $U$ et $B$ est très voisine (tabl. 2). Les teneurs en énergie métabolisable et PDI ont été estimées à partir de la composition chimique des aliments et de ses ingrédients, respectivement, en accord avec les méthodes proposées par l'INRA (1978). Pour le calcul de la PDI, la teneur en PDIE de la paille traitée à la soude a été établie sur la base d'une digestibilité de sa MO de 65,2 p. 100, déterminée par incubation in vitro (TILley \& TERRY, 1963) avec 4 aliments témoins de digestibilité connue, tandis que la teneur en PDIN du tourteau de soja traité à la chaleur a été évaluée à partir de sa solubilité corrigée, estimée par l'équation exposée par VERITE pour les matières premières (INRA, 1978). 
Tableau 2

Composition chimique des aliments (p. 100 de MS).

Feed chemical composition (DM, p. 100).

\begin{tabular}{|c|c|c|}
\hline & \multicolumn{2}{|c|}{$\begin{array}{l}\text { Source d'ANPI } \\
N P N \text { compound }\end{array}$} \\
\hline & $\begin{array}{l}\text { Urée } \\
\text { Urea }\end{array}$ & $\begin{array}{l}\text { Biuret } \\
\text { Biuret }\end{array}$ \\
\hline Matière organique - Organic matter ... & 92,20 & 92,20 \\
\hline Matières azotées totales - Crude protein & 15,36 & 15,34 \\
\hline Extrait éthéré - Ether extract $\ldots \ldots \ldots$ & 1,79 & 1,87 \\
\hline Cellulose brute - Crude fibre . . . . . . . . & 14,46 & 14,64 \\
\hline «Acid detergent fibre $»-$ Acid detergent fibre $\ldots$. & 18,96 & 19,17 \\
\hline $\begin{array}{l}\text { Energie métabolisable (MCal/kgMS) } \ldots \ldots \ldots \ldots \\
\text { Metabolizable energy (McallkgDM) }\end{array}$ & 2,77 & 2,77 \\
\hline PDIN $(g / k g M S)-(g / k g D M) \ldots \ldots \ldots \ldots$ & 88,50 & 88,30 \\
\hline PDIE $(g / \mathrm{kgMS})-(g / k g D M) \ldots \ldots \ldots \ldots$ & 87,50 & 87,20 \\
\hline
\end{tabular}

\section{Prélèvements}

Les mesures de digestibilité et de bilan azoté ont été réalisées durant une période de 5 jours. Sur les quantités totales quotidiennes de fèces et d'urine excrétées, des échantillons équivalents à $10 \mathrm{p} .100 \mathrm{du}$ poids ou du volume ont été prélevés ; ils ont été ensuite conservés à $-20^{\circ} \mathrm{C}$ avant d'être mélangés et analysés.

L'activité cellulolytique du rumen a été évaluée en incubant, pendant 48 heures, dans le sac ventral du rumen de chaque agneau, 3 sachets en toile de nylon $(8 \times 14 \mathrm{~cm}$ de dimensions et $60 \mu \mathrm{m}$ de diamètre de pore), contenant $4 \mathrm{~g}$ de paille traitée à la soude (identique à celle des aliments), broyée à la grille de $1 \mathrm{~mm}$. Les résidus non digérés ont été séchés à $105^{\circ} \mathrm{C}$ pendant 24 heures; les dosages des teneurs en ADF avant et après incubation ruminale permettent d'en calculer le pourcentage de disparition.

Les paramètres des fermentations ruminales ont été mesurés sur une période de 24 heures, aux temps suivants après le repas : $1,2,4,6,8,10$ et 12 heures d'une part et 1,2 et 4 heures d'autre part pour les régimes à 2 et 6 repas/jour, respectivement. Pour la détermination de la concentration en ammoniac et en $\mathrm{AGV}$, on a prélevé, pour chaque type de dosage, deux échantillons de $5 \mathrm{ml}$ de jus du rumen qui ont été mélangés respectivement avec $5 \mathrm{ml}$ de $\mathrm{HCl} 0,5 \mathrm{~N}$ ou $1 \mathrm{ml}$ de $\mathrm{H}_{3} \mathrm{PO}_{4} 3 \mathrm{M}$ et conservés à $-20^{\circ} \mathrm{C}$. Simultanément, on a relevé le $\mathrm{pH}$ du jus du rumen avec un $\mathrm{pH}$-mètre digital.

\section{Analyses}

La composition chimique des aliments, des fèces et de l'urine a été déterminée suivant les techniques de l'A.O.A.C. (1975) et la teneur en ADF d'après la méthode de Goering \& Van Soest (1970). L'ammoniac a été dosé selon la technique de 
Conway (1957). Les concentrations en AGV ont été déterminées par chromatographie en phase gazeuse selon la méthode exposée par JouANy (1982).

Les résultats des mesures de $\mathrm{pH}$ et des concentrations en ammoniac et AGV ont été comparés (Test de Student) de façon indépendante pour chaque fréquence d'alimentation et heure post-prandiale. Les résultats des contrôles de digestibilité et des bilans azotés ont été appréciés par analyse de variance, en accord avec un modèle factoriel ( 2 aliments $\times 2$ fréquences de distribution d'aliment), en considérant l'effet dû aux animaux comme hiérarchisé à l'effet aliment; les moyennes entre traitements ont été comparées par le test de la moindre différence significative.

\section{Résultats}

\section{A. Paramètres de la fermentation dans le rumen}

L'évolution du $\mathrm{pH}$ du jus du rumen (fig. 1) n'a pas été très différente entre les deux aliments; cependant, on observe un comportement différent en fonction de la fréquence des repas. Ainsi, avec le régime à 2 repas/jour, les valeurs du $\mathrm{pH}$ avec l'aliment $U$ sont plus faibles qu'avec l'aliment B ; pour 6 repas/jour c'est le contraire ; toutefois, les différences ne sont significatives qu'immédiatement avant la distribution des repas $(\mathrm{P}<0,05)$.

L'augmentation du nombre journalier de repas entraîne une élévation des valeurs des minima du $\mathrm{pH}$ avec les deux aliments (de 5,43 et 5,50 à 5,78 et 5,89 avec les aliments $U$ et $B$, respectivement) et de même, une forte réduction des fluctuations de ce paramètre. Ce dernier effet a été plus net avec l'aliment $B$, et conduit alors à une relative stabilité du $\mathrm{pH}$ lors de 6 repas/jour, s'expliquant par les faibles variations enregistrées des concentrations en ammoniac et en AGV (fig. 1 et 2).

Les concentrations en ammoniac évoluent différemment selon l'aliment (fig. 1). Avec le rythme à 2 repas/jour, elles sont plus élevées avec l'aliment U qu'avec l'aliment $\mathrm{B}$ aux temps de $1,2(\mathrm{P}<0,01)$ et $4(\mathrm{p}<0,05)$ heures après le repas, mais, pour les temps de $10(\mathrm{P}<0,05)$ et $12(\mathrm{P}<0,01)$ heures l'effet contraire a été observé. Avec 6 repas/jour, les concentrations obtenues avec l'aliment $U$ ont été nettement supérieures à celles atteintes avec l'aliment $B$ pendant la première moitié de la période postprandiale, la différence étant significative $(\mathrm{P}<0,05) 1$ heure après le repas.

Ces résultats montrent que l'emploi du biuret par rapport à l'urée entraîne une moindre ammoniogénèse post-prandiale réduisant ainsi les accumulations de ce métabolite dans le jus du rumen.

Par comparaison avec 2 repas/jour, le rythme de 6 repas/jour provoque une réduction nette des fluctuations des concentrations d'ammoniac, avec une forte diminution des valeurs maximales. 


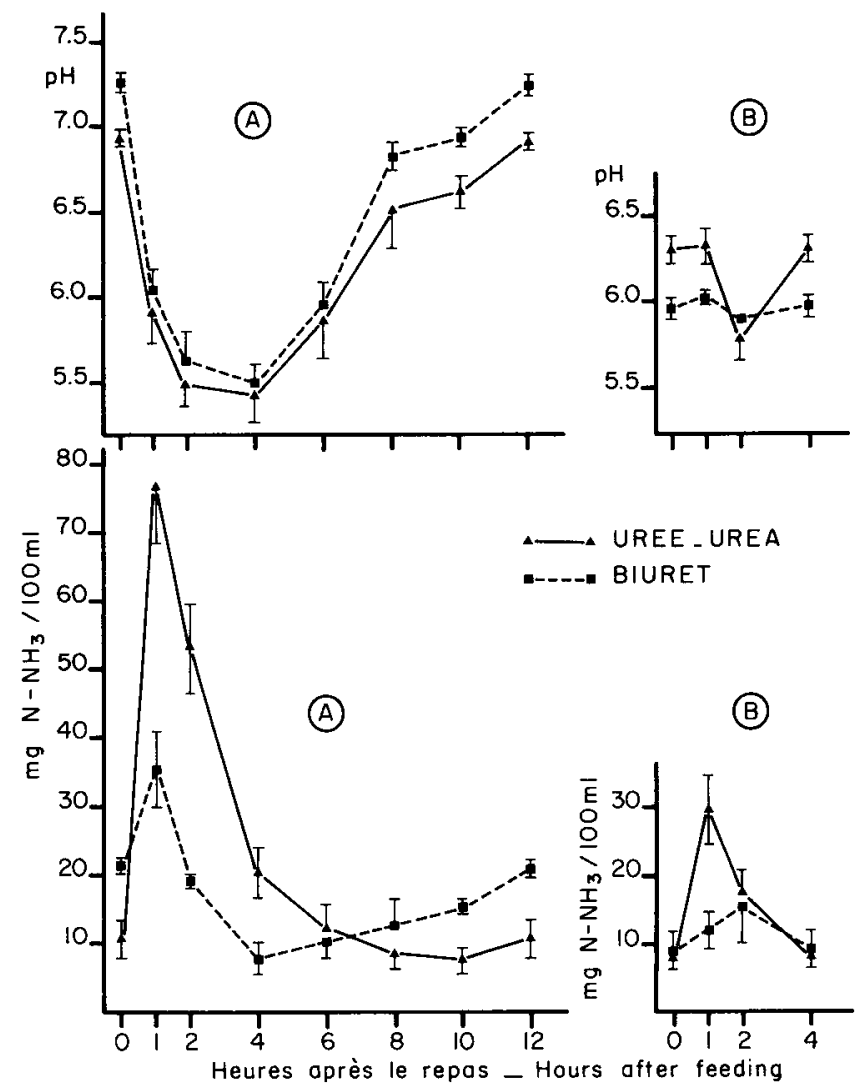

Fig. 1

Evolution du $\mathrm{pH}$ et des concentrations en ammoniac du jus du rumen en distribuant la ration en $2(A)$ ou 6 (B) repas/jour. (Les barres verticales représentent les écarts-types).

Variation of ruminal $\mathrm{pH}$ and ammonia concentrations according to feeding frequency: 2 (A) or 6 (B) meals/day. (The standard deviations are represented by vertical bars).

Les concentrations en AGV ont été très variables d'un animal à l'autre. Ceci explique que l'on n'ait pas pu trouver de différence significative entre aliments, que ce soit pour les concentrations totales en AGV (fig. 2), pour les pourcentages molaires moyens des acides acétique, propionique et butyrique, ou pour le rapport acétique/ propionique (tabl. 3). L'augmentation du nombre quotidien de repas a entraîné également une réduction très importante de la variation des concentrations totales en AGV; elle a produit aussi, avec l'aliment B, des élévations et diminutions apparentes des pourcentages des acides acétique et propionique, respectivement. Par contre, avec l'aliment $U$, la multiplication des repas n'a pas eu d'effet sur le type de fermentation. 


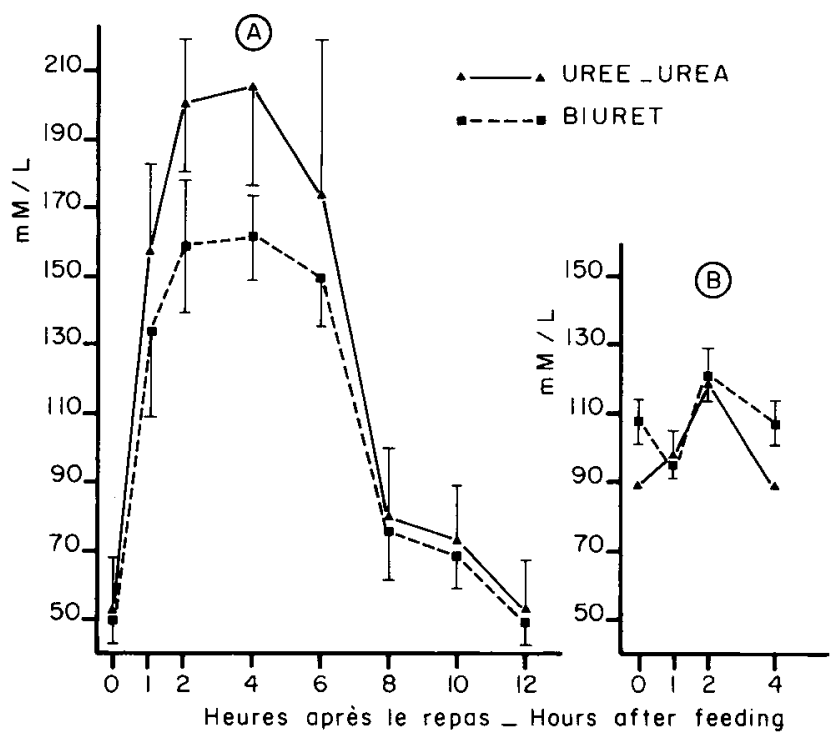

FIG 2

Evolution des concentrations en acides gras volatils $d u$ jus $d u$ rumen en distribuant la ration en 2 (A) ou 6 (B) repas/jour. (Les barres verticales représentent les écarts-types).

Variation of ruminal volatile fatty acid concentrations according to feeding frequency: $2(A)$ or $6(B)$ meals/day. (The standard deviations are represented by vertical bars).

TABLEAU 3

Pourcentages molaires moyens des acides acétique $\left(C_{2}\right)$, propionique $\left(C_{3}\right)$ et butyrique $\left(C_{4}\right)$ dans les $A G V$ totaux et rapport acétiquelpropionique $\left(C_{2} / C_{3}\right)$ (moyenne \pm écart-type).

Mean molar percentages of acetic $\left(C_{2}\right)$, propionic $\left(C_{3}\right)$ and butyric $\left(C_{+}\right)$acids, and acetic/propionic ratio $\left(C_{2} / C_{3}\right)$ (mean and standard deviation).

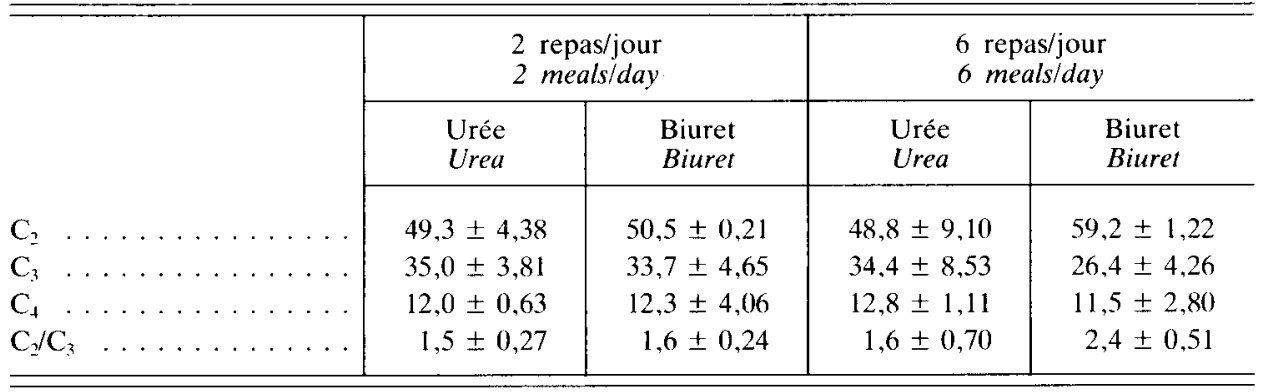

B. Utilisation digestive et bilan azoté

Dans le tableau 4 sont présentés les coefficients d'utilisation digestive apparents des principaux constituants chimiques de la ration. On y indique également le pourcentage 
d'ADF disparu à partir des échantillons de paille placés dans des sachets de nylon et incubés dans le rumen, ainsi que les résultats des bilans azotés.

\section{TABLEAU 4}

Coefficients d'utilisation digestive (CUD), activité cellulolytique dans le rumen et bilan azoté (moyenne \pm écart-type).

Digestibility coefficients, ruminal cellulolytic activity and nitrogen balances (mean and standard deviation).

\begin{tabular}{|c|c|c|c|c|}
\hline \multirow[b]{3}{*}{$\begin{array}{l}\text { CUD apparents (p. 100) } \\
\text { Apparent digestibility (p. 100) }\end{array}$} & \multicolumn{2}{|c|}{$\begin{array}{l}2 \text { repas/jour } \\
2 \text { meals/day }\end{array}$} & \multicolumn{2}{|c|}{$\begin{array}{l}6 \text { repas/jour } \\
6 \text { meals/day }\end{array}$} \\
\hline & $\begin{array}{l}\text { Urée } \\
\text { Urea }\end{array}$ & $\begin{array}{l}\text { Biuret } \\
\text { Biuret }\end{array}$ & $\begin{array}{l}\text { Urée } \\
\text { Urea }\end{array}$ & $\begin{array}{l}\text { Biuret } \\
\text { Biuret }\end{array}$ \\
\hline & & & & \\
\hline $\begin{array}{l}\text { Matière organique } \ldots \ldots \ldots \\
\text { Organic matter }\end{array}$ & $68,9 \pm 0,97$ & $68,7 \pm 2,04$ & $71,2 \pm 4,18$ & $70,4 \pm 2,75$ \\
\hline $\begin{array}{l}\text { — Acid detergent fibre } » \ldots . . \\
\text { Acid detergent fibre }\end{array}$ & $29,0 \pm 1,38^{a}$ & $28,0 \pm 0,57^{\mathrm{a}}$ & $34,1 \pm 3,53^{b}$ & $34,5 \pm 2,91^{\mathrm{b}}$ \\
\hline $\begin{array}{l}\text { Matières azotées totales .... } \\
\text { Crude protein }\end{array}$ & $79,4 \pm 1,80$ & $78,5 \pm 0,66$ & $79,7 \pm 0,06$ & $77,5 \pm 1,09$ \\
\hline $\begin{array}{l}\text { Activité cellulolytique }{ }^{(1)} \text { p. } 100 \ldots \\
\text { Cellulolytic activity }\end{array}$ & $31,1 \pm 1,16^{\mathrm{c}}$ & $35,6 \pm 2,38^{\text {cd }}$ & $36,1 \pm 2,42^{\mathrm{d}}$ & $54,5 \pm 2,97^{\mathrm{e}}$ \\
\hline $\begin{array}{l}\text { Bilan azoté }{ }^{(2)} \mathrm{g} / \mathrm{Al} / \mathrm{j} \quad \ldots \ldots \\
\text { Nitrogen balance } g / F d / d\end{array}$ & $3,8 \pm 0,59^{\mathrm{c}}$ & $5,4 \pm 0,60^{\mathrm{d}}$ & $5,9 \pm 0,63^{\mathrm{d}}$ & $5,3 \pm 0,48^{d}$ \\
\hline
\end{tabular}

(1) «Acid detergent fibre » de paille disparue des sachets (p. 100) après un séjour dans le rumen de 48 heures Acid detergent fibre of straw disappeared from nylon bags (p. 100) after a residence time in the rumen of 48 hours.

(2) g $\mathrm{N}$ retenu/animal/jour - $g \mathrm{~N}$ retained/animal/day.

a, b : Les valeurs d'une même ligne avec différents indices sont significativement différentes (P $<0,01)$ - Values on the same line with different supercripts are significantly different $(P<.01)$.

c, d, e : Les valeurs d'une même ligne avec différents indices sont significativement différentes $(P<0,05)$ Values on the same line with different supercripts are significantly different $(P<.05)$.

Aucun effet dû aux sources d'ANPI n'a été mis en évidence sur les coefficients d'utilisation digestive; par contre, on a noté un accroissement $(P<0,01)$ assez fort $(20,3$ p. 100$)$ de la digestibilité de l'ADF avec l'augmentation du nombre de repas par jour. Etant donné l'âge relativement avancé des animaux (5 mois), on peut penser que la faible différence d’âge entre les deux expériences ( 15 jours) n'a pas pu influencer considérablement ces résultats. La digestibilité plus élevée des constituants pariétaux pourrait donc être attribuée au changement de fréquence d'alimentation.

L'analyse de la variance des coefficients de disparition in sacco de l'ADF de la paille a montré l'existence d'une interaction $(P<0,01)$ entre la source d'ANPI et la fréquence d'alimentation, qu'on peut attribuer à l'activité cellulolytique très supérieure induite par l'aliment B quand celui-ci a été distribué en 6 repas/jour. On a constaté aussi, que la digestion de l'ADF est plus forte avec le biuret par rapport à l'urée $(P<0,01)$ et avec la fréquence supérieure des repas $(P<0,01)$, avec un net effet cumulatif de ces deux paramètres. 
Par ailleurs, l'analyse de la variance des bilans azotés met en évidence une interaction $(P<0,05)$ entre la source d'ANPI et la fréquence d'alimentation ; ainsi, les bilans d'azote augmentent en fonction du nombre de repas/jour avec l'aliment $U$ mais pas avec l'aliment $B$.

\section{Discussion}

\section{A. Paramètres de la fermentation dans le rumen}

L'atténuation de la baisse du pH observée avec l'augmentation du nombre journalier de repas peut être expliquée par l'établissement d'une fermentation plus uniforme dans le rumen, comme cela a été suggéré par Kaufmann, Hagemeister \& Dirksen (1979).

Les moindres accumulations d'ammoniac dans le rumen avec l'aliment $B$ résultent vraisemblablement de la plus faible solubilité et vitesse d'hydrolyse du biuret par rapport à l'urée (FonNesBeck, KEARL \& Harris, 1975).

En relation avec l'aliment $B$, il faut signaler, en plus, que son utilisation ruminale doit être améliorée du fait que le biuret employé a une teneur relativement élevée en urée $(18,7$ p. 100). Ceci a pu être observé avec le rythme de 2 repas/jour pour lequel la concentration en ammoniac a diminué rapidement (après le pic obtenu à 1 heure), probablement en raison de son utilisation par les microorganismes du rumen, jusqu'à atteindre des concentrations comprises dans l'intervalle de $5-10 \mathrm{mg} \mathrm{N}-\mathrm{NH}_{3} / 100 \mathrm{ml}$ nécessaires pour une croissance microbienne optimale (Hume, Morr \& Somers, 1970 ; Satter \& Siyter, 1974 ; Okorie, Buttery \& Lewis, 1977). Si on avait employé un biuret d'une pureté plus élevée, on aurait pu constater une moindre accumulation initiale dammoniac et, par conséquent, des concentrations postérieures plus faibles et donc limitantes pour la croissance microbienne. Cette observation confirme les conclusions de Nikolic et al. (1979) indiquant que la vitesse de production de l'ammoniac à partir du biuret peut être, dans certains cas, insuffisante pour satisfaire les besoins azotés de la microflore du rumen.

\section{B. Utilisation digestive et bilans d'azote}

L'accroissement de la digestibilité de l'ADF avec l'augmentation de la fréquence d'alimentation est en accord avec les résultats exposés par KaufmanN, Hagemeister \& DikKSEN (1979). Il doit être attribué à une activité plus intense de la flore cellulolytique du rumen comme cela est confirmé par l'élévation parallèle des coefficients de disparition " in sacco" de l'ADF de la paille broyée. Cette cellulolyse supérieure a pu être favorisée par laugmentation des valeurs minimales du $\mathrm{pH}$, car il est bien connu que la baisse du $\mathrm{pH}$ entraine une réduction de la densité des bactéries cellulolytiques (Osbourn et al., 1970 ; Journet, 1971 ; Stewark, 1977).

Les différences observées entre les aliments, concernant les coefficients de disparition "in sacco" de l'ADF de la paille broyée avec le rythme de 6 repas/ jour, indiquent que l'aliment $B$ doit induire une cellulolyse plus active, qui serait en accord avec l'élévation apparente du rapport acétique/propionique. Cependant, ces diffërences 
ne se sont pas traduites pour ce qui concerne la digestibilité de l'ADF de la ration. Cette contradiction entre les résultats « in sacco " et " in vivo " pourrait être expliquée par une digestion plus intense des constituants pariétaux de l'aliment $U$ dans le caecum et le côlon. qui compenserait la moindre dégradation dans le rumen. Il est possible également que l'estimation du pouvoir cellulolytique pour un temps de séjour fixe de 48 heures ne soit pas représentative de la véritable activité cellulolytique correspondant aux temps de séjour réels des constituants pariétaux des rations dans le rumen.

Les différences constatées entre les aliments, dans les rétentions azotées avec 2 repas/jour, ainsi que l'élévation de ces rétentions avec l'aliment $U$ quand la fréquence d'alimentation est passée de 2 à 6 repas/jour, démontrent la moindre efficacité de l'urée par rapport au biuret, lors d'une distribution peu fractionnée. Cette moindre utilisation est peut-être due à des fuites plus notables d'ammoniac du rumen, en raison des niveaux de concentration atteints. Cependant, ces fuites possibles ont dû se produire principalement, par la sortie d'ammoniac avec le flux de digesta, car, en raison des valeurs basses du $\mathrm{pH}$ (inférieures à 6,5) associées aux fortes concentrations d'ammoniac, l'absorption d'ammoniac au niveau de l'épithélium du rumen n'a pas dû être très marquée (ChAlmers, JafFray \& WhiTe, 1971). Par conséquent, on peut s'attendre à ce que la différence entre les deux sources d'ANPI essayées, quand elles sont consommées en un nombre faible de repas, serait sans doute encore supérieure avec des rations dont la digestion ruminale s'accompagne de valeurs de $\mathrm{pH}$ plus hautes.

\section{Conclusion}

En conclusion de cette expérimentation, on peut retenir que l'augmentation du nombre de repas par jour a un effet positif sur la digestibilité de l'« acid detergent fibre » des rations ainsi que sur l'efficacité de l'urée, vraisemblablement en raison, dans ce dernier cas, d'une réduction des fuites d'ammoniac du rumen.

Ces résultats montrent d'autre part, que l'utilisation du biuret, par comparaison à l'urée, doit présenter des avantages pour les régimes utilisant des doses élevées d'ANPI en distributions peu fractionnées, alors que l'efficacité de ces deux produits doit rester semblable lorsque les repas sont nombreux.

Une teneur relativement élevée en urée dans les produits du biuret semble avoir un effet positif sur l'emploi des rations de haute concentration énergétique, pour lesquelles la vitesse de libération d'ammoniac à partir de produits d'un biuret plus pur pourrait ne pas être suffisante pour couvrir les besoins d'azote fermentescible de la population microbienne du rumen.

Reçu en juillet 1986.

Accepté en janvier 1987. 


\section{Summary}

Comparative utilization of urea and biuret in diets offered 2 or 6 times a day in sheep

Utilization of two compound feeds containing urea (U) or biuret (B) and offered as 2 or 6 meals per day was measured. after an adaptation period of 5 weeks, in two groups of three lambs fitted with rumen cannulas.

Apparent digestibility coefficients, nitrogen balances, $\mathrm{pH}$ and concentrations of ammonia and volatile fatty acids in rumen liquor were determined as well as the disappearance in sacco of the acid detergent fibre (ADF) of samples of ground straw.

Concentrations of ammonia in rumen liquor were significantly lower $(P<0.05)$ with diet $B$ than with diet $U$. Increasing the number of meals from 2 to 6 reduced peak concentrations of ammonia more markedly with diet $U$ than with diet $B$. Likewise, the increase in the number of meals improved $(P<0.05)$ nitrogen balance with diet $U(3.84$ and $5.93 \mathrm{~g}$ Nitrogen retained/animal/ day with 2 and 6 meals/day, respectively) whereas this effect was not observed with diet $B(5.42$ and $5.31 \mathrm{~g}$ Nitrogen retained/animal/day).

Apparent digestibility coefficients of organic matter, crude protein and ADF were not different except that of ADF which was lower $(P<0.01)$ with $2(28.3$ p. 100) than with 6 meals/ day $(34.3 \mathrm{p} .100)$. Moreover, with 6 meals/day ADF disappearance in the rumen was lower $(\mathrm{P}<0.01)$ with diet $U(36.1$ p. 100$)$ than with $\operatorname{diet} B(54.5$ p. 100).

Key words: Urea, biuret, feeding frequency, sheep.

\section{Références bibliographiques}

A.O.A.C., 1975. Official methods of analysis (12th Ed.). Association of Official Agricultural Chemist, Washington, D.C., 957 p.

Campbell J.C., Loosli J.K., Warner R.G., JASAKI J., 1963. Utilization of biuret by ruminants. J. Anim. Sci, 22, 139-145.

Chalmers M.I., JafFray A.E., White F., 1971. Movements of ammonia following intraruminal administration of urea or casein. Proc. Nutr. Soc., 30, 7-17.

Clark R., Barret E.L., Kellerman J.H., 1963. A comparison between nitrogen retention from biuret and urea on a low roughage diet. J.S. African Vet. Med. Ass., 34, 419-425.

Clemens E.T., Johnson R.R., 1973. Influence of dietary nitrogen source, concentrate level and biuret level in sheep on the adaptation of rumen microorganisms to biuret as a non-protein nitrogen source. J. Nutr., 103, 1406-1413.

Conway E.J., 1957. Microdiffusion analysis and volumetric error. 4th Ed. Crosby Lockwood. London, $468 \mathrm{p}$.

Fonnesbeck P.V., Kearl L.C., Harris L.E., 1975. Feed grade biuret as a protein replacement for ruminants. J. Anim. Sci., 40, 1150 - i 184.

Goeking H.K., VAN SoEst P.J., 1970. Forage fiber analysis. U.S.D.A. Agric. Handbook N 379. $20 \mathrm{p}$.

Hume I.D., MoIr R.J., Somers M., 1970. Synthesis of microbial protein in the rumen : 1 . Influence of the level of nitrogen intake. Aust. J. Agric. Res., 21, 283-296.

INRA, 1978. Alimentation des ruminants. Ed. INRA. Publications, Route de Saint-Cyr, 78000 Versailles, $621 \mathrm{p}$.

JouAny J.P., 1982. Volatile fatty acid and alcohol determination in digestive contents, silage juices, bacterial cultures and anaerobic fermentor contents. Sciences des Aliments, 2, 131-144.

Journet M., 1971. Influence du mode de présentation des fourrages déshydratés sur la digestion dans le rumen et le taux butyreux des vaches. Ann. Biol. Anim. Biochim. Biophys., 11, $296-297$.

Kaufmann W., Hagemeister H., Dirksen G., 1979. Adaptation to changes in dietary composition, level and frequency of feeding. In : Digestive Physiology and Metabolism in Ruminants. Ed. Ruckebusch Y., Thivend P., 587-603. M.T.P. Press Ltd, Lancaster, Grande-Bretagne. 
Morr R.J., Somers M., Bray A.C., 1967. Utilization of dietary sulphur and nitrogen by ruminants. Sulphur Inst. J., 3, 15-21.

Nikolic J.A., Pavličević D., Zeremski D., Negovanovic D., 1979. Adaptation to diets containing significant amounts of non-protein nitrogen. In: Digestive Physiology and Metabolism in Ruminants. Ed. Ruckebusch Y., Thivend P., 603-620, M.T.P. Press Ltd, Lancaster, GrandeBretagne.

OKorie A.U., Buttery P.J., Lewis D., 1977. Ammonia concentration and protein synthesis in the rumen. Proc. Nutr. Soc., 36, 38 A.

Osbourn D.F., Terry R.A., Cammel S.B., Outen G.F., 1970. Some effects of feeding supplement of maize meal and sodium bicarbonate upon the digestion of forage cellulose by sheep. Proc. Nutr. Soc., 29, 12 A.

Satter L.D., Slyter L.L., 1974. Effects of ammonia concentration on rumen microbial protein production in vitro. Br. J. Nutr., 32, 199-208.

SingHal K.K., Mudgal V.D., 1980. Biuret, a non-protein nitrogen source for ruminants. $J$. Nuclear Agric. Biol., 9, 64-70.

Singhal K.K., Mudgal V.D., 1984. Biuret and urea in the ration of milk goats : a full lactation study. Indian J. Anim. Sci, 54, 180-189.

Stewark C.S. 1977. Factors affecting the cellulolytic activity of rumen contents. Appl. Environ. Microbiol., 33, 497-502.

Tilley J.M.A., Terry R.A., 1963. A two stage technique for the in vitro digestion of forage crops. J. Br. Grassld. Soc., 18, 104-111.

WoLrer R., L'azote non protéique dans l'alimentation des ruminants. 1. Mode de valorisation et tolérance à l'urée. Rev. de Méd. Vét., 125, 761-779. 